Zusammenfassung der Dissertation mit dem Titel

\title{
„Die Individualsanktionen des Sicherheitsrates der Vereinten Nationen und die Grund- und Menschenrechte aus der Europäischen Menschenrechtskonvention"
}

Dissertation vorgelegt von Layla Kristina Jaber

Erstgutachter: Prof. Dr. Martin Borowski

Zweitgutachter: Prof. Dr. Seibert-Fohr

Institut für Staatsrecht, Verfassungslehre und Rechtsphilosophie 


\section{Einleitung}

Die vorliegende Arbeit befasst sich mit dem Verhältnis der Individualsanktionen des Sicherheitsrates der Vereinten Nationen $\mathrm{zu}$ den prozessualen Gewährleistungen der Europäischen Menschenrechtskonvention (EMRK). Die sich daraus ergebende Pflichtenkollision wird umfassend untersucht und eine Lösung für die Einrichtung einer gerichtlichen Kontrolle der nationalen Umsetzungsakte dieser Sanktionen erarbeitet.

Einige der Herausforderungen des modernen Völkerrechts sind die asymmetrischen Gefahren, die von Terroristen oder anderen Individuen ausgehen, die international organisiert sind, aber keinem Staat zugerechnet werden können. Darüber hinaus sind auch ehemalige Regierungsmitglieder von Staaten in den Fokus des Völkerrechts geraten, da sie als Urheber von Kriegen und Krisen zur Verantwortung gezogen und von weiteren Tätigkeiten abgehalten werden sollen. Die Gemengelage der verschiedenen Rechtsgüter und Interessen ist in diesem Kontext sehr komplex, da mit neuen und intensiveren Maßnahmen gegen internationale Gefahren zum Schutz der Sicherheit, Eingriffe in die individuelle Freiheit und damit in die Grund- und Menschenrechte einhergehen. Um diesen Gefahren entgegenzuwirken, erlässt der Sicherheitsrat seit nunmehr zwei Jahrzehnten Individualsanktionen auf der Grundlage des Kapitel VII der UN-Charta.

Das Völkerrecht, das regionale Recht und das nationale Recht verweben sich zusehends miteinander und bilden somit ein komplexes Mehrebenensystem aus. Die Ordnungsfunktion des Völkerrechts ist nicht mehr seine einzige Funktion. Es kam schließlich zur Rechtsvereinheitlichung, angetrieben von einer Judikatur vielfältiger internationaler Gerichte und einer Vielzahl internationaler Verträge. Des Weiteren wurden neuartige Rechtsakte durch internationale Organisationen erlassen, die sich gegen Individuen richten. Sie haben dazu geführt, dass sich die verschiedenen Ebenen immer stärker miteinander verflochten haben.

Dadurch erfuhr das Völkerrecht einen Bedeutungszuwachs, mit dem auch eine größere Verantwortung der Organe internationaler Organisationen einherging, der sie indes nicht immer gerecht werden. Mit der Rechtsetzungstätigkeit der exekutiven Organe internationaler Organisationen, mit denen individuelle Rechtspositionen betroffen werden, haben sich Berührungspunkte mit dem Recht der Staatenverantwortlichkeit ergeben, da nicht mehr nur ein Autor eines Rechtsaktes oder einer Handlung in Betracht kommt, sondern mehrere Akteure beteiligt sind. Dies rührt daher, dass eine internationale Organisation grundsätzlich keine durchgreifenden Rechtsakte gegen Individuen erlassen kann, sondern diese Rechtsakte erst 
durch die Umsetzung und Durchführung der Staaten Wirkung gegenüber dem Einzelnen entfalten können.

Erlässt der Sicherheitsrat der Vereinten Nationen bindende Resolutionen nach dem Kapitel VII UN-Charta, die die Mitgliedstaaten in ihre nationalen Rechtsordnungen umsetzen, kommen bei einer Verletzung von individuellen Rechten sowohl die Vereinten Nationen als auch die Mitgliedstaaten als Verantwortliche in Betracht. Diese Vielschichtigkeit stellt die internationale Gemeinschaft vor neue Herausforderungen.

\section{Problemstellung}

Die vorliegende Arbeit illustriert, wie vielschichtig die Probleme im Zusammenhang mit den Individualsanktionen des Sicherheitsrates im Mehrebenensystem sind. Die präventiven Maßnahmen mit quasi-legislativem Charakter, die aufgrund ihrer völkerrechtlichen Verbindlichkeit für die Mitgliedstaaten der Vereinten Nationen zur Folge haben, dass Individuen durch die Mitgliedstaaten sanktioniert werden, sind aus grund- und menschenrechtlicher Sicht höchst problematisch. Denn aufgrund der undurchsichtigen Ausgestaltung des Sanktionsverfahrens in Sanktionskomitees des Sicherheitsrates ist die ,Schuld‘ des Betroffenen nicht zweifelsfrei bewiesen. Auch die Effektivität der Maßnahmen kann bezweifelt werden, da trotz des nunmehr seit fast zwei Jahrzehnten bestehenden AlQuaida Sanktionsregimes, internationale terroristische Vereinigungen weltweit weiterhin aktiv sind.

Diese Individualsanktionen sind ein sehr anschauliches Beispiel für die enge Verknüpfung der verschiedenen Ebenen im internationalen Kontext des Mehrebenensystems. Die bindenden Resolutionen des Sicherheitsrates werden durch verschiedene Gesetzgeber, seien sie national oder regional, in das nationale bzw. regionale Recht umgesetzt. Und diese nationalen Umsetzungsrechtsakte werden dann wiederum durch nationale, regionale und internationale Grund- und Menschenrechte tangiert. Infolgedessen kollidieren die Verpflichtungen der Mitgliedstaaten bei den Individualsanktionen des Sicherheitsrates gerade wegen der oben beschriebenen zweifelhaften Sanktionspraxis des Sicherheitsrates.

In dieser Dissertation wird ein Konzept herausgearbeitet, mit dem die Staaten des Europarates, der Europäische Gerichtshof für Menschenrechte (EGMR) und auch die nationalen Gerichte arbeiten können, um die Pflichtenkollisionen im Rahmen der Individualsanktionen des Sicherheitsrates mit dem europäischen Menschenrechtsschutz aufzulösen und um den 
fehlenden Zugang zu einem Gericht gegen die Individualsanktionen des Sicherheitsrates durch die Eröffnung des Rechtsweges vor nationalen Gerichten auszugleichen.

Ausgangspunkte sind die Fälle $N a d a^{1}$ und Al-Dulimi ${ }^{2}$. Die Große Kammer des EGMR entschied in der Sache Nada anders als seinerzeit die Kammer in der Sache Al-Dulimi in Bezug auf die Begründung der Zuständigkeit des EGMR. Daher ist die genauere Untersuchung der Verantwortlichkeit der Mitgliedstaaten für die Umsetzung der Individualsanktionen des Sicherheitsrates von zentralem Interesse. Die Große Kammer, der die Sache Al-Dulimi nach Art. 43 EMRK vorgelegt wurde, hat in der Begründung der Zuständigkeit im Sinne der Sache Nada entschieden. Die Große Kammer prüfte die nationalen Umsetzungsakte am Maßstab der EMRK und konnte daher die Schweiz wegen einer Verletzung der EMRK verurteilen.

Da die Rechtsprechung des EGMR im Zentrum dieser Arbeit steht, wird ein Vorschlag unterbreitet, um einen gerechten Ausgleich zwischen den jeweiligen staatlichen Verpflichtungen zu finden, ohne das Gefüge des Völkerrechts zu destabilisieren. Dabei wird die Zuständigkeit des EGMR und der nationalen Gerichte für die Rechtsakte im Mehrebenensystem begründet. Denn es sind nicht nur die staatlichen Verpflichtungen, die sich aus der UN-Charta und der EMRK ergeben, vor allem sind die Einzelschicksale derjenigen zu berücksichtigen, die sich einer Sanktionierung durch den Sicherheitsrat ausgesetzt sehen, ohne dafür durch ein internationales oder nationales Gericht verurteilt worden zu sein. Daher wird in dieser Arbeit untersucht, ob das Sanktionsverfahren des Sicherheitsrates der Vereinten Nationen die Standards eines fairen Verfahrens und des effektiven Rechtsschutzes erfüllen. Aber auch die Vereinbarkeit mit Gewährleistungen, wie die auf ein Familienleben, die Bewegungsfreiheit, das Eigentum und die freie Meinungsäußerung, die durch die Individualsanktionen betroffen sein können, muss berücksichtigt werden.

Die vorliegende Arbeit entwickelt ein Modell für einen effektiven Schutz der Grund- und Menschenrechte innerhalb des Systems der EMRK in Europa. Denn dem EGMR und den mitgliedstaatlichen Gerichten wird eine Begründungsgrundlage geliefert, auf dessen Basis sie sich zur Kontrolle der nationalen Umsetzungsrechtsakte der Individualsanktionen des Sicherheitsrates für zuständig erklären können. In der Folge können sie diese Umsetzungsrechtsakte am Grundrechtestandard der EMRK messen. All dies hat zum Ziel, den

\footnotetext{
${ }^{1}$ EGMR (GK), Urteil vom 12. September 2012, n 10593/08 - Nada gg. Schweiz.

2 EGMR, Urteil vom 26. November 2013, $\mathrm{n}^{\circ}$ 5809/08 - Al-Dulimi and Montana Managment Inc. gg. Schweiz; EGMR (GK), Urteil vom 21. Juni 2016, $n^{\circ}$ 5809/08 - Al-Dulimi and Montana Managment Inc. gg. Schweiz.
} 
Grundrechtsschutz im Mehrebenensystem, bestehend aus den Vereinten Nationen, den Regionalorganisationen und den nationalen Rechtsordnungen, zu stärken.

Die in dieser Untersuchung im Zentrum stehende Konstellation unterscheidet sich von derjenigen der Mitgliedstaaten der Europäischen Union, da diese durch die Übernahme der Umsetzung der Resolutionen im Bereich der Individualsanktionen des Sicherheitsrates durch die EU dahingehend entlastet sind. Auch hat der EuGH in seiner Rechtsprechung seine Zuständigkeit zur Kontrolle der europäischen Umsetzungsrechtsakte bereits geklärt ${ }^{3}$ und mit einer anderen Begründungslinie, basierend auf der Autonomie der Rechtsordnung der EU, gearbeitet. Der EuGH misst seither in ständiger Rechtsprechung die Umsetzungsrechtsakte der EU am Maßstab der Charta der Grundrechte der EU. Daher steht die Kontrolle der europäischen Umsetzungsakte durch den EuGH nicht im Zentrum der vorliegenden Untersuchung.

\section{Methodik und Gliederung}

Zu Beginn dieser Arbeit (1. Kapitel) werden die derzeitigen Maßnahmen des Sicherheitsrates im Bereich der Individualsanktionen vorgestellt. Dazu wird die Entwicklung der Sanktionen des Sicherheitsrates auf der Basis von Kapitel VII UN-Charta skizziert, die sich von auf Staaten gerichteten Maßnahmen zu solchen entwickelt haben, die auch Individuen anvisieren können. Anschließend (2. Kapitel) folgt eine theoretische Untersuchung der Probleme im Mehrebenensystem, da sich die Individualsanktionen des Sicherheitsrates dadurch auszeichnen, dass sich verschiedene Rechtsebenen miteinander verknüpfen. Daher werden die verschiedenen Ansichten über das Verhältnis des nationalen Rechts zum Völkerrecht und das Verhältnis internationaler Rechtsregime zueinander beleuchtet. Die Entscheidungen dieser Gerichte stehen im Vordergrund, weil diese Fragen von der nationalen und internationalen Rechtsprechung behandelt wurden. Von besonderem Interesse für diese Arbeit ist dabei die Rechtsprechung des EGMR.

Der Theorienstreit über das Verhältnis der Normen im Mehrebenensystem wird hier relevant: Zum Verhältnis des nationalen Rechts zum Völkerrecht stehen sich die dualistische und die monistische Theorie gegenüber. Zum Verhältnis internationaler Teilrechtsregime konkurrieren der Pluralismus und der Konstitutionalismus.

Darüber hinaus gibt es verschiedene Werkzeuge, um Normkollisionen und Normkonflikte im Mehrebenensystem zu lösen, welche in dieser Arbeit dargestellt und kritisch gewürdigt werden. Dazu gehören sowohl Konfliktvermeidungsregeln wie die systemische und harmonisierende

\footnotetext{
${ }^{3}$ EuGH, Urteil vom 3. September 2008, Kadi gg. Rat und Komission, C-402/05 P und C-415/05 P, ECLI:EU:C:2007:61.
} 
Auslegung und die diversen, Solange-Formeln“ bzw. equialent-protection-Formeln aus der Rechtsprechung nationaler und internationaler Gerichte, als auch Konfliktlösungsregeln, wie die Kollisionsnorm in Art. 103 UN-Charta.

Art. 103 UN-Charta spielt eine besondere Rolle, da er nach seinem Wortlaut einen grundsätzlichen Vorrang der Verpflichtungen aus der UN-Charta vor allen anderen vertraglichen Verpflichtungen der Mitgliedstaaten der Vereinten Nationen anordnet. Damit scheint diese Kollisionsnorm grundsätzlich der Umsetzung und Durchführung der Sanktionen gegen Individuen einen Vorrang vor den grund- und menschenrechtlichen Gewährleistungen aus internationalen Verträgen - insbesondere den Zugang zu einem Gericht - einzuräumen. Diese strenge Sichtweise führte dazu, dass einige Gerichte sich für unzuständig im Hinblick auf die Kontrolle der nationalen Umsetzungsmaßnahmen am Maßstab von Grund- und Menschenrechten erklärten.

Da nach der in dieser Arbeit vertretenen Auffassung das Verhältnis der internationalen Ebenen zueinander derzeit nach einer pluralistischen Sichtweise verstanden werden muss und eine Hierarchie im Völkerrecht mit einem absoluten Vorrang der UN-Charta nicht gilt, bedeutet dies für die Individualsanktionen des Sicherheitsrat das Folgende: das regionale oder das nationale Gericht, das mit einem Einzelfall befasst ist, prüft mithilfe der Auslegungsparameter insbesondere mit der harmonisierenden Auslegung - die Vereinbarkeit der Sanktionen mit den Grundrechten. Diese Befugnis leitet sich aus dem formellen Subsidiaritätsgrundsatz ab. Dabei bringt das Gericht im Wege der Abwägung das Ziel der internationalen Sicherheit und die Grundrechte des Betroffenen miteinander in Einklang.

Eine Konstitutionalisierung des Völkerrechts im Sinne einer verfassten Rechtsordnung ist bisher nicht zu beobachten, auch wenn sich in einigen Teilbereichen sehr ausdifferenzierte Systeme mit eigenen Gerichtsbarkeiten entwickelt haben. Insbesondere die UN-Charta ist keine Weltverfassung, da sie sich lediglich auf die Wahrung des internationalen Friedens und der Sicherheit konzentriert und dazu nach dem Willen der Staaten gegründet wurde. Daher führt auch die Kollisionsnorm aus Art. 103 UN-Charta nicht zu einem absoluten Vorrang aller Verpflichtungen aus der UN-Charta vor dem sonstigen Völkerrecht, sondern sie ist eng auszulegen.

Anschließend (3. Kapitel) steht die Rolle des Sicherheitsrates im Zentrum der Untersuchung, da er der primäre Autor der Individualsanktionen ist, die die Mitgliedstaaten umsetzen und anwenden. Dazu werden seine Aufgaben und Funktionen, die ihm die Mitgliedstaaten vor allem durch das Kapitel VII UN-Charta übertragen haben, untersucht und im Hinblick darauf 
hinterfragt, ob die aktuelle Sanktionspraxis mit der UN-Charta noch vereinbar ist. Der Schwerpunkt liegt dabei auf den Grenzen des Handelns des Sicherheitsrates.

Dabei wird festgestellt, dass der Sicherheitsrat in den vergangenen Jahren seine Befugnisse und Tätigkeiten nach Kapitel VII der UN-Charta immer stärker ausgeweitet hat. Dennoch gibt es Grenzen für das Handeln des Sicherheitsrates, die in dieser Dissertation herausgearbeitet werden. Es handelt sich dabei um die Rule of Law, die internationalen Menschenrechte - wie sie sich aus dem ius cogens, der UN-Charta und dem allgemeinen Völkerrecht ergeben - und den Verhältnismäßigkeitsgrundsatz. Im Rahmen der Verhältnismäßigkeit der Maßnahmen, muss der Sicherheitsrat das zu erreichende Ziel seiner Maßnahme mit dem Eingriff in die Grundrechte der Betroffenen abwägen.

Die Bindung des Sicherheitsrates an die internationalen Grund- und Menschenrechte und die internationale Rule of Law unterstreicht umso mehr das Ergebnis dieser Arbeit, als dass die nationalen und regionalen Gerichte, mangels Rechtsschutz auf der internationalen Ebene, den Betroffenen der Individualsanktionen den entsprechenden Rechtsschutz gewährleisten müssen. Aufgrund des immer stärker auch auf das Individuum ausgerichteten Völkerrechts und der zentralen Rolle des Menschenrechtsschutzes im Völkerrecht ist die Verwehrung des Zugangs zu einem Gericht nicht zu rechtfertigen.

Durch das undurchsichtige Verfahren der Auflistung und Sanktionierung der Personen durch das Sanktionskomitee des Sicherheitsrates oder die zuständigen nationalen Stellen kommt es bspw. zu Verwechselungen, die durch unabhängige Untersuchungen aufgeklärt werden müssen. Aufgrund der Bindung des Sicherheitsrates an die internationalen Grund- und Menschenrechte muss es möglich sein, dass sich die Individuen, die sich zu Unrecht sanktioniert sehen, vor einer unabhängigen Stelle gegen die Sanktionen wehren können. Des Weiteren muss der Sicherheitsrat beim Erlass der Sanktionen auf der Grundlage des Kapitel VII UN-Charta den Grundsatz der Verhältnismäßigkeit berücksichtigen, der von ihm verlangt, nur die Maßnahmen zu erlassen, die zum Schutz der Gemeinschaftsgüter erforderlich sind und mit der geringsten Beeinträchtigung ihrer Rechte einhergehen.

Abgerundet werden diese Untersuchungen mit der Analyse der Verantwortlichkeit der Beteiligten der Sanktionsverfahren, also des Sicherheitsrates und der Mitgliedstaaten, und der Kontrollmöglichkeit des Handelns des Sicherheitsrates durch verschiedene Akteure. Diese Fragen sind schließlich dann von Relevanz, wenn die Maßnahmen des Sicherheitsrates, so wie sie durch die Mitgliedstaaten umgesetzt werden, gegen das für sie bindende Recht verstoßen und die Rechtpositionen der betroffenen Personen verletzt. 
Abschließend (4. Kapitel) folgt die Untersuchung der gerichtlichen Zuständigkeiten in den Mitgliedstaaten der EMRK für die Kontrolle der nationalen Umsetzungsrechtsakte der Sanktionen. Dazu wird die Rechtsprechung des EGMR genauer durchleuchtet, die sich mit der Zuständigkeit des EGMR zur Kontrolle von nationalen Umsetzungsrechtsakten von Rechtsakten internationaler Organisationen befasst. Dies umfasst die Rechtsprechung in Bezug auf die EU und in Bezug auf andere europäische Organisationen. Dabei steht der Fokus auf den Urteilen zu Individualsanktionen des Sicherheitsrates.

\section{Zentrale Thesen}

Die in dieser Arbeit ausgearbeitete Rekonstruktion der Zuständigkeit des EGMR und der nationalen Gerichte für die Kontrolle der nationalen Umsetzungsrechtsakte der Individualsanktionen des Sicherheitsrates legt die damit zusammenhängenden Fragen im Mehrebenensystem dar. Die Beleuchtung der Praxis des Sicherheitsrates und seiner Kompetenzen und der Rechtsprechung des EGMR im Kontext der Individualsanktionen zeigt sich als ein bottom-up-approach.

Der Lösungsvorschlag begründet die Zuständigkeit der mitgliedstaatlichen Gerichte und des EGMR zur Kontrolle der nationalen Umsetzungsakte mit dem Argument, dass die Vereinten Nationen derzeit und in absehbarer Zeit nicht in der Lage sein werden, einen den internationalen und regionalen Menschenrechtsstandards genügenden direkten Rechtsschutz gegen die Individualsanktionen des Sicherheitsrates zu gewähren. Aus diesem Grund wird die Kompetenz der unteren Ebenen im Mehrebenensystem herangezogen, die einen deutlich besseren Grundrechtsschutz leisten können, wie sich an der EMRK und der Rechtsprechung des EGMR - und natürlich auch der Charta der Grundrechte der EU - zeigt. Diese Lösung führt im Ergebnis zu einer starken Gewichtsverlagerung zugunsten der europäischen Staaten in Bezug auf die gerichtliche Kontrolle im Mehrebenensystem und wird in Europa, wegen der Bindung der Staaten an die EMRK, zu überzeugenden Ergebnissen führen.

Durch diese Untersuchung wird das Sanktionsregime gegen Individuen des Sicherheitsrates auf seine Vereinbarkeit mit der EMRK untersucht und eine Kontrollbefugnis des EGMR und der nationalen Gerichte hergeleitet. Da sich die Befugnisse des Sicherheitsrates in diesem Bereich stark erweitert haben, gleichzeitig aber der Rechtsschutz auf der Ebene der Vereinten Nationen nicht verbessert wurde, ist eine solche Rekonstruktion zum Schutz der individuellen Grundund Menschenrechte unbedingt erforderlich. 
Die Staaten haben bei der Umsetzung der direkten Individualsanktionen des Sicherheitsrats keinen Umsetzungsspielraum, im Gegensatz zu den indirekten Sanktionen. Die Prämisse, nach der der EGMR in den Rechtssachen Nada und Al-Dulimi seine Zuständigkeit entwickelt hat, ist daher sehr widersprüchlich. Da die Lösung des EGMR nicht befriedigend ist, folgt eine Rekonstruktion der Zuständigkeit des EGMR und damit auch der nationalen Gerichte nach dem Grundsatz der (formellen) Subsidiarität.

Dieses Prinzip bewirkt, dass auch im Kontext des Völkerrechts immer dann die unterste oder die dem Individuum am nächsten liegende Einrichtung zuständig ist. Dies hat, in Bezug auf den Menschenrechtsschutz eine immense Bedeutung, da der effektive Schutz der individuellen Menschenrechte ein Anliegen aller Staaten und auch der Vereinten Nationen ist.

Argumente für die Anwendung des Subsidiaritätsgrundsatzes als Begründung für die Zuständigkeit der regionalen und nationalen Gerichte sind zum einen die materielle und formelle Subsidiarität aus der UN-Charta, dem Völkerrecht, aber auch der EU, der EMRK und dem Recht föderaler Staaten. Zum anderen sind die Resolutionen des Sicherheitsrates mit einem Makel (mangelnder Rechtsschutz) versehen, den die Staaten verpflichtet sind auszugleichen: Dies ergibt sich aus den Grundsätzen der Transparenz, der Fairness, dem Effektivitätserfordernis und dem Grundsatz der Organisationstreue. Abe auch aus ihrer eigenen Bindung an internationale Grund- und Menschenrechte.

Um einen effektiven Grundrechtsschutz im Bereich der Individualsanktionen gewährleisten zu können, sollten die nationalen Gerichte und der EGMR folgendermaßen vorgehen:

1. Bei den indirekten Sanktionen wird aufgrund des Umsetzungsspielraums das nationale Umsetzungsgesetz dem Staat zugerechnet. Konsequenterweise erfolgt die Kontrolle durch regionale und nationale Gerichte.

2. Bei den direkten Sanktionen ohne Umsetzungsspielraum für die umsetzenden Staaten gibt es grundsätzlich keine Kontrolle durch die nationalen und regionalen Gerichte aufgrund des Vorrangs der Pflichten aus der UN-Charta gem. Art. 103, es sei denn, die Anwendung der equivalent-protection-Formel auf das System der Vereinten Nation ergibt, dass der dortige Grundrechtsschutz das Schutzniveau der EMRK unterschreitet. Dieser Lösungsansatz wird aus dem Subsidiaritätsgrundsatz hergeleitet.

Der Subsidiaritätsgrundsatz ist ein zusätzlicher Begründungsansatz für die Übertragung der equivalent-protection-Formel auf die Kontrolle der nationalen Umsetzungsakte, die die Sanktionen des Sicherheitsrates ins nationale Recht umgesetzt haben. Denn eine Verantwortlichkeit der Staaten für den Umsetzungsakt nach dem Recht der 
Staatenverantwortlichkeit besteht auch bei Resolutionen ohne Umsetzungsspielraum. Haben die nationalen Stellen bei der Umsetzung den Zugang zum Gericht nicht eingerichtet, kann dies durch die Gerichte selbst erfolgen.

Eine Zuständigkeit der Gerichte lässt sich mithilfe des Subsidiaritätsgrundsatzes herleiten, ohne den Sanktionsmechanismus des Sicherheitsrates grundsätzlich in Frage zu stellen, denn auch das Völkerrecht muss es aushalten, dass mit Hoheitsgewalt ausgeführte Akte kontrolliert werden können. Die Gewährleistung des Rechtswegs ergänzt damit das Sanktionsregime des Sicherheitsrates und führt gar zu einer Stärkung und Konsolidierung der Individualsanktionen. Aufgrund der fehlenden Rechtsschutzmöglichkeiten auf der Ebene der Vereinten Nationen kann mithilfe des Grundsatzes der Subsidiarität und einer engen Auslegung des Art. 103 UNCharta die Zuständigkeit der nationalen und regionalen Gerichte hergeleitet werden. Der Maßstab für die Mitgliedstaaten ist die EMRK, die in Art. 6 und 13 EMRK ein faires Verfahren und eine effektive Beschwerde gewährleistet. Für die Individualsanktionen des Sicherheitsrates gelten dabei die Maßstäbe des strafrechtlichen Verfahrens, da die Sanktionen der Schwere nach strafrechtlichen Maßnahmen gleichkommen. Der Grundsatz der Subsidiarität führt daher im Ergebnis dazu, dass die nationalen Umsetzungsakte der Individualsanktionen des Sicherheitsrates am Maßstab der EMRK kontrolliert werden können.

Der Ansatz dieser Arbeit wurde für die Fälle herausgearbeitet, die nicht schon dem Unionsrecht unterfallen. Im Gegensatz zur Rechtsprechung des EuGH, der die europäischen Umsetzungsrechtsakte der Resolutionen des Sicherheitsrates am Maßstab der Unionsgrundrechte prüft und sich zur Begründung auf die ,Autonomie des Unionsrechts“ beruft, kann sich der EGMR im Recht der EMRK auf dieses Argument nicht stützen.

Aus diesem Grund wurde in dieser Arbeit ein anderer Ansatz gesucht, um für die im Ergebnis begrüßenswerte Rechtsprechung des EGMR eine nachvollziehbare und den europäischen Menschenrechtsschutz wertschätzende Argumentationslinie bereitzustellen. Auf der Grundlage des Subsidiaritätsgrundsatzes kann daher eine konsequente gerichtliche Kontrolle der nationalen Umsetzungsakte durchgeführt werden, bei der im Wege der Abwägung unter Berücksichtigung der Einzelfallgerechtigkeit, die sich gegenüberstehenden Rechtsgüter miteinander in Einklang gebracht werden.

\section{Ausblick}

Auch wenn diese Ausarbeitung lediglich ein Lösungsvorschlag für das europäische Menschenrechtsschutzsystem bietet, kann sie dennoch als Blaupause für andere regionale Menschenrechtsschutzsysteme herangezogen werden. Insbesondere die Übertragung auf das 
System der Amerikanischen Menschenrechtskonvention (AMRK) ist denkbar, da der Interamerikanische Gerichtshof für Menschenrechte ebenso dafür zuständig ist, die nationalen Rechtsakte und damit auch die Umsetzungsrechtsakte der Individualsanktionen des Sicherheitsrates am Maßstab der AMRK zu messen.

Global betrachtet führt dieser Ansatz dazu, dass in vielen Staaten der Welt die Umsetzungsrechtsakte der Resolutionen nicht an einem Maßstab, der der EMRK gleichwertig wäre, geprüft werden, da sie über einen anderen Grundrechtestandard verfügen. In vielen Staaten der Welt ist der individuelle Grundrechtsschutz leider noch unzureichend ausgestaltet, sodass nicht an eine weltweit einheitliche Gewährleistung von effektivem Rechtsschutz gegen Individualsanktionen zu denken ist. Diese Problematik, sowie die Frage der Übertragbarkeit dieser These sind von großem Interesse, werden aber in dieser Arbeit keine Berücksichtigung finden. Dies soll anderen Untersuchungen vorbehalten bleiben.

Würde ein adäquater Grundrechtsschutz auf der Ebene der Vereinten Nationen eingerichtet, der den Betroffenen von Individualsanktionen, die direkt durch den Sicherheitsrat oder indirekt durch die Mitgliedstaaten auf einer Liste aufgeführt wurden, einen Rechtsschutzmechanismus an die Hand gibt, um die durch die Staaten ausgeführten wirtschaftlichen Sanktionen auszusetzen, wäre ein weltweit einheitlicher Grundrechtestandard gewährleistet. Dies würde dazu führen, dass die Betroffenen direkt auf der Ebene der Vereinten Nationen gegen die Sanktionen vorgehen könnten. Eine entsprechende Entscheidung könnte dann Präjudizwirkung für die nationalen Rechtsordnungen entfalten, die die Sanktionen umgesetzt haben, sodass die Staaten automatisch mit der Beendigung der Sanktionen reagieren müssten. Dies würde es den Betroffenen ermöglichen alle nationalen Umsetzungsrechtsakte der Sanktionen infrage zu stellen, ohne in jedem Staat aufs Neue den langwierigen Rechtsweg vor den nationalen Gerichten durchlaufen zu müssen. Da aber eine derartige Verbesserung des Grundrechtsschutzes auf der Ebene des Sicherheitsrates und den Vereinten Nationen nicht abzusehen ist, soll bis dahin die in dieser Untersuchung vorgeschlagene Lösung weiterhelfen. 